\title{
Critical Sensitivity and Trans-scale Fluctuations in Catastrophic Rupture
}

\author{
Meng Fen Xia, ${ }^{1,2}$ Yu Jie Wei, ${ }^{1}$ Fu Jiu Ke, ${ }^{1,3}$ \\ and YI LONG BAI ${ }^{1}$
}

\begin{abstract}
Rupture in the heterogeneous crust appears to be a catastrophe transition. Catastrophic rupture sensitively depends on the details of heterogeneity and stress transfer on multiple scales. These are difficult to identify and deal with. As a result, the threshold of earthquake-like rupture presents uncertainty. This may be the root of the difficulty of earthquake prediction. Based on a coupled pattern mapping model, we represent critical sensitivity and trans-scale fluctuations associated with catastrophic rupture. Critical sensitivity means that a system may become significantly sensitive near catastrophe transition. Trans-scale fluctuations mean that the level of stress fluctuations increases strongly and the spatial scale of stress and damage fluctuations evolves from the mesoscopic heterogeneity scale to the macroscopic scale as the catastrophe regime is approached. The underlying mechanism behind critical sensitivity and trans-scale fluctuations is the coupling effect between heterogeneity and dynamical nonlinearity. Such features may provide clues for prediction of catastrophic rupture, like material failure and great earthquakes. Critical sensitivity may be the physical mechanism underlying a promising earthquake forecasting method, the load-unload response ratio (LURR).
\end{abstract}

Key words: Critical sensitivity, trans-scale fluctuations, catastrophe transition, sample-specificity, heterogeneous media.

\section{Introduction}

As the humanity enters the New Millennium, it inherits the great achievements in the sciences and technology along with a traditional bandage, one of the greatest societal concerns, the problem of earthquake prediction. This subject has attracted considerable interest, and knowledge about earthquakes has significantly advanced in the past century. Nevertheless the self-similarity of earthquakes (GUTENBERG et al., 1944) and self-organized criticality (SOC) (BAK et al., 1987, 1988; BAK, 1994) aroused heated discussion about the predictability of earthquakes. Are we really on ground where the stresses are near failure everywhere and at all time? Most scientists

\footnotetext{
${ }^{1}$ State Key Laboratory of Nonlinear Mechanics (LNM), Institute of Mechanics, Chinese Academy of Sciences, Beijing 100080, China. E-mail: weiyj@Inm.imech.ac.cn, baiyl@lnm.imech.ac.cn

${ }^{2}$ Department of Physics, Peking University, Beijing 100871, China. E-mail: xiam@lnm.imech.ac.cn

${ }^{3}$ Department of Applied Physics, Beijing University of Aeronautics and Astronautics, Beijing 100083, China. E-mail: kefj@lnm.imech.ac.cn
} 
agree on another viewpoint, that earthquake prediction remains a contemporary difficulty given the current knowledge (KNOPOFF, 2000; Wyss et al., 1997). Clearly, further study of the physics of preparation for catastrophic rupture is required.

A series of recent works suggest that earthquake might depend sensitively on the details of heterogeneous structure and stress transfer in the earth's crust. (DiODATI et al., 1991; LOCKNER et al., 1991, 1992; GARCIMARTIN et al., 1997; WANG et al., 1998; Lu et al., 1998; Heimpel, 1997; SteIn, 1999; CuRRAN et al., 1997). This is quite similar to rupture in heterogeneous brittle media. Rupture appears to be a catastrophe transition (BAI et al., 1994; WEI et al., 2000) and the threshold of catastrophe shows uncertainty (XIA et al., 1997; XIA et al., 2000). It is insufficient to represent the rupture of disordered heterogeneous media by only macroscopically averaged properties (SAHimi et al., 1993; MEAKin, 1991; IBNABDElJalil et al., 1997; CURTIN, 1997).

A large earthquake may be considered as a local catastrophic rupture in the earth's crust. The main underlying mechanism behind the complex behaviors of earthquakes and failure of disordered brittle materials might be attributed to the coupling between disordered heterogeneity on multiple scales (BEN-ZION et al., 2000) and dynamical nonlinearity during nonequilibrium evolution (BAI et al., 1994; CURRAN et al., 1997; WeI et al., 2000). In order to identify clues for prediction of earthquakes and material failure, a possible strategy is to explore general features of catastrophic rupture in heterogeneous brittle media.

Coupling effects between disordered heterogeneity on the mesoscopic scale and dynamical nonlinearity are so complex that direct experimental observations or theoretical conclusions are quite difficult. Even for numerical simulations, some reasonable simplification of physical concepts is a necessity. Recently, we have examined a coupled pattern mapping model (XIA et al., 2000; WEI et al., 2000) similar to the well-known fiber-bundle model (Coleman, 1958; Daniels, 1945). However our model takes the coupling effects between mesoscopic disordered heterogeneity and dynamical nonlinearity due to stress redistribution into account. We found that such a model can reproduce distinctive features of rupture in complex heterogeneous media.

Notably, the model displays the catastrophe transition (BAI et al., 1994; WEI et al., 2000) and sample-specificity (XIA et al., 1997, 2000), namely macroscopic uncertainty of observed rupture behavior. This is one of the roots for the difficulty in rupture prediction. We report here that the catastrophe transition presents some general features: critical sensitivity and trans-scale fluctuations. The critical sensitivity implies that the system may become significantly sensitive near the catastrophe transition. Trans-scale fluctuations refer to fluctuations which, at the catastrophe threshold, may be enhanced strongly and accordingly, the spatial scales of stress and damage fluctuations increase rapidly from the mesoscopic heterogeneity scale to the macroscopic scale. These general features provide insight into the essence of the catastrophe transition, and may provide clues for prediction of catastrophic ruptures, such as great earthquakes. 
Section 2 briefly reviews the coupled pattern mapping model. Section 3 presents the evolution of the model behaviour. Critical sensitivity and trans-scale fluctuations are considered in sections 4 and 5, respectively. Section 6 contains a summary of the results and discussion.

\section{Brief Review of Model}

The model (BAI et al., 1994; XIA et al., 1997, 2000; WEI et al., 2000) is a periodic lattice consisting of $N$ mesoscopic units. Mesoscopic heterogeneity is modelled by assigning randomly an initial strength $\sigma_{c i}$ to each unit $i$ with $\left\{\sigma_{c i}\right\}$ given by distribution function $h\left(\sigma_{c}\right)$.

Mesoscopically, the system is specified by the damage pattern $X=\left\{x_{i}, i=\right.$ $1,2, \ldots, N\}$, the stress pattern $\Sigma=\left\{\sigma_{i}, i=1,2, \ldots, N\right\}$, and the initial strength pattern $\Sigma_{c}=\left\{\sigma_{c i}, i=1,2, \ldots, N\right\}$, where an intact or broken unit $i$ is denoted by $x_{i}=0$ or 1 , respectively. $\sigma_{i}$ is the stress on unit $i$ and $\sigma_{c i}$ is the initial strength of unit $i$. Macroscopic parameters are the damage fraction,

$$
p=\frac{1}{N} \sum_{i=0}^{N} x_{i},
$$

and the nominal stress,

$$
\sigma_{0}=\frac{1}{N} \sum_{i=0}^{N} \sigma_{i}
$$

Samples with identical $h\left(\sigma_{c}\right)$ are considered to be identical macroscopically, although they are different from sample to sample mesoscopically due to disordered mesoheterogeneity. In the following calculations, we choose $h\left(\sigma_{c}\right)$ as a Weibull distribution function with a mean of 1 and a modulus, $m_{c}=2$.

The pattern dynamics is defined by iterations of mappings between the coupled patterns $X, \Sigma$ and $\Sigma_{c}$. The evolution of damage pattern $X$ is determined by the stress pattern $\Sigma$ and the strength pattern $\Sigma_{c}$, according to a mesoscopic failure condition. It is simply assumed that all units with $\sigma_{i} \geq \sigma_{c i}$ break simultaneously. The strength pattern $\Sigma_{c}$ varies with damage pattern $X$ as $\sigma_{c i}\left(1-x_{i}\right)$. This means that a broken unit loses strength, and no longer supports any stress. The stress pattern $\Sigma$ is determined from the damage pattern $X$ according to a stress redistribution (SRD) rule, and it is assumed to be independent of history. It is convenient to represent the SRD rule with respect to a state with uniform stress $\left(\sigma_{i}=\sigma_{0}\right)$.

In order to examine the effects of stress fluctuations, we considered various SRD rules as follows:

(1) Global mean field (GMF) model: The nominal stress $\sigma_{0}$ of broken units is shared by all intact units uniformly. 
(2) Local mean stress concentration (LMSC) model: The nominal stress of a broken cluster is uniformly transferred to its two neighboring intact regions of size $\delta$.

(3) Cluster mean field (CMF) model: The nominal stress of a broken cluster is equally redistributed to its two neighboring intact clusters and the stress within an intact cluster is uniform.

The LMSC and CMF models display stress fluctuations, whereas the GMF model is without stress fluctuations. The SRD rule represents the main dynamical nonlinearity in the model.

The evolution of the system is controlled by external loading, i.e., the nominal stress $\sigma_{0}$ increases from $\sigma_{0}=0$ to a failure threshold $\left(\sigma_{0}=\sigma_{0 f}\right)$. We will consider quasi-static loading as a standard process: the nominal stress increment $\Delta \sigma_{0}$ is computed each loading step as the minimum increment necessary to break at least one unit.

\section{Catastrophe Transition}

We represent the evolution of a system by considering the time series of energy release $\Delta E$. The energy release $\Delta E$ is calculated as a summation of the initial stored elastic energy of broken units in a mapping, or loading step $\Delta \sigma_{0}$. For simplicity, the dimensionless elastic modulus is assumed to be 2 and is identical for all units, and the stored elastic energy of a unit can be written as $\sigma_{i}{ }^{2}$. Because the damage fraction $p$ increases monotonously, the time series of $\Delta E$ can be shown as a plot of $\Delta E$ versus $p$.

Figure 1 shows time series of energy release $\Delta E$ for various SRD models. A distinct common feature is that the evolution presents a catastrophe transition at $p_{c}$, which corresponds to a threshold of nominal stress $\sigma_{0 f}$. For a specified nominal stress below the threshold $\left(\sigma_{0}<\sigma_{0 f}\right)$, the evolution remains in a globally stable (GS) mode with mesoscopic damage accumulation. At the threshold $\left(\sigma_{0}=\sigma_{0 f}\right)$ however, the system falls into a condition of self-sustained catastrophic failure $(\mathrm{CF})$, the main rupture appears and the system evolves to entire failure $(p=1)$ eventually. Such an evolution-induced mode transition demonstrates a general behavior of failure in heterogeneous brittle media. This behavior is called evolution induced catastrophe (EIC).

Generally speaking, rupture prediction is concerned particularly with the catastrophe transition and main rupture. Unfortunately, it is found that the threshold of catastrophe transition $\left(\sigma_{0 f}\right)$ shows uncertainty. Thus, it is impossible to predict catastrophic rupture in terms of a few macroscopic parameters such as the damage fraction $p$, the nominal stress $\sigma_{0}$ and parameters defining the properties of the strength distribution (e.g., modulus $m_{c}$, when the Weibull distribution function is applied). Figure 2 shows an ensemble distribution of catastrophe transition threshold $\left(\sigma_{0 f}\right)$ for samples which are identical macroscopically. For the GMF model without stress fluctuations, all samples exhibit identical threshold $\sigma_{0 f}$ determined by strength 


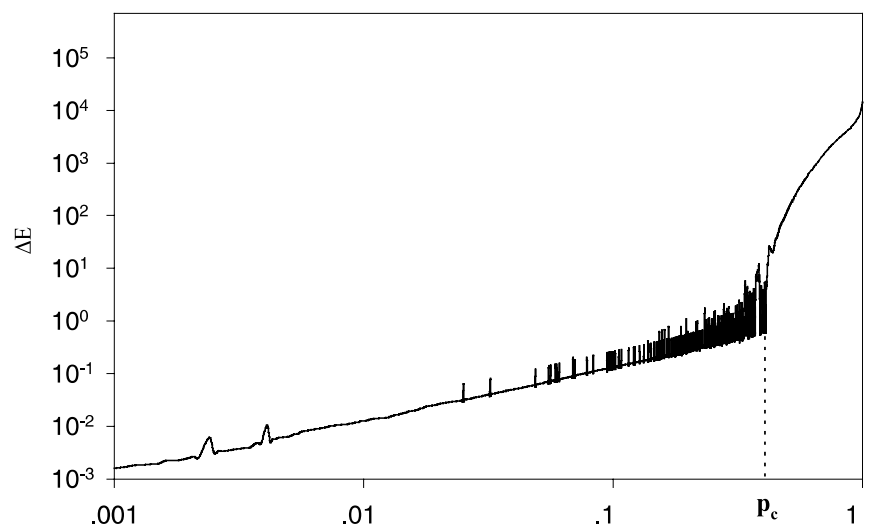

a)

p

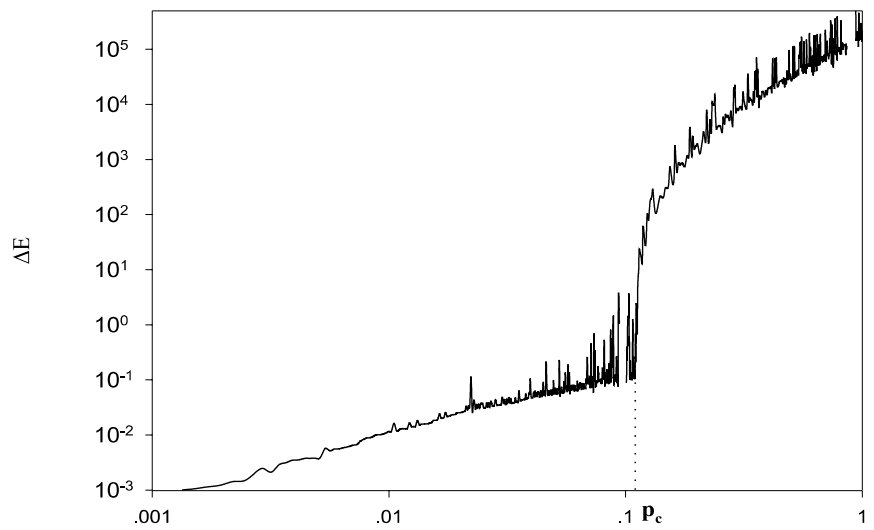

b)

$\mathbf{p}$

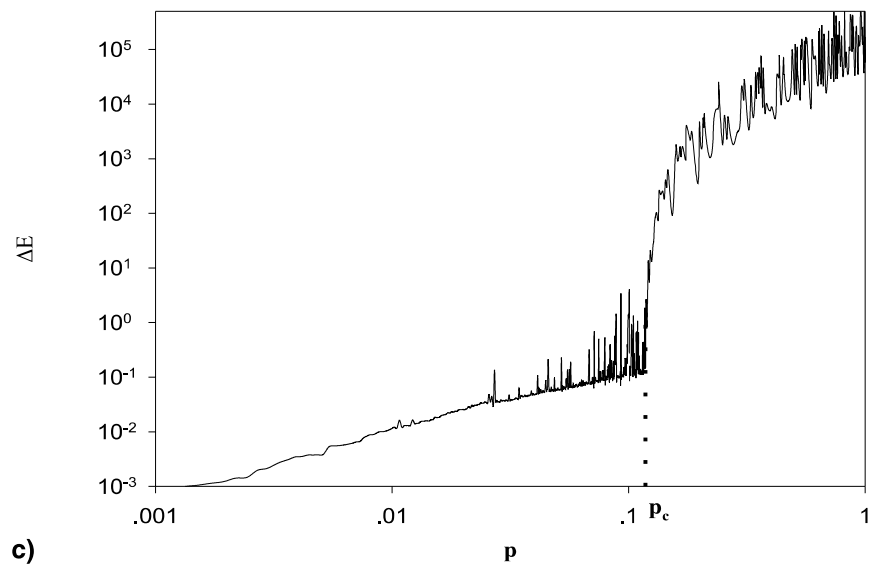

Figure 1

The energy release $\Delta E$ versus damage fraction $p$ for a mapping with $N=10,000$. (a) GMF model, (b) LMSC model with $\delta=5$, (c) CMF model. 
distribution function $h\left(\sigma_{c}\right)$. In this case rupture prediction is deterministic, based only upon macroscopic parameters. However, for models with stress fluctuations, LMSC and CMF, the threshold $\sigma_{0 f}$ shows diversity, i.e., the threshold is different from sample to sample for macroscopical identical samples. Such a behavior is called sample-specificity (XIA et al., 1997, 2000). Such diversity of macroscopic failure strength has been reported by SAHIMI et al. (1993) and BAI et al. (1994). Samplespecificity leads to macroscopic uncertainty, and rupture prediction becomes impossible based upon macroscopic parameters alone.

In order to circumvent this obstacle to rupture prediction, a possible strategy is to explore some universal features of the catastrophe transition in search of clues of use in rupture prediction.

A well-known general feature of threshold systems is that the size-distribution of events follows a power law. This has been observed in both material failure (DIODATI et al., 1991; GARCIMARTIN et al., 1997; Lu et al., 1998) and earthquakes (as evidenced by the empirical Gutenberg-Richter relation) (GUTENBERG and RichTER, 1944). Our model also displays power-law event size-distribution. Figure 3 gives the statistics $N(\Delta E)$ of energy release $\Delta E$ calculated for each loading step. The log-log plot follows a rather respectable straight line ranging about $1.5 \sim 2$ decades. In this model, the power-law size-distribution is mainly attributed to events prior to the catastrophe transition. The exponent ( $b$ value) is not universal. A power law is suggestive of a dynamical system near criticality, corresponding with the catastrophe

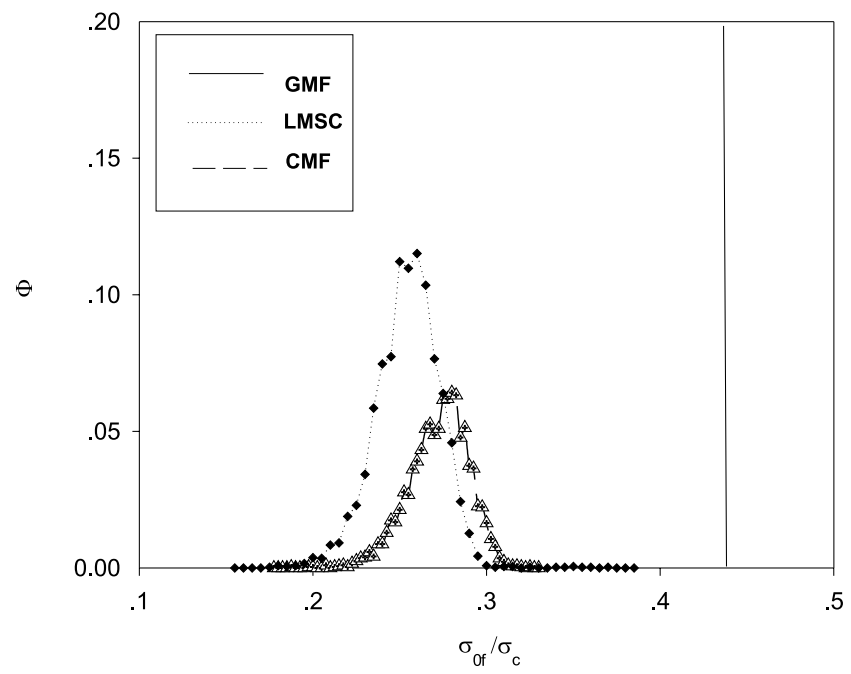

Figure 2

Ensemble distribution of the catastrophe transition threshold $\sigma_{0 f}$ for samples which are identical macroscopically. 10,000 samples with $N=8000$ were examined. The solid line represents the GMF model.

The dotted line represents the LMSC model and the dashed line represents the CMF model. 


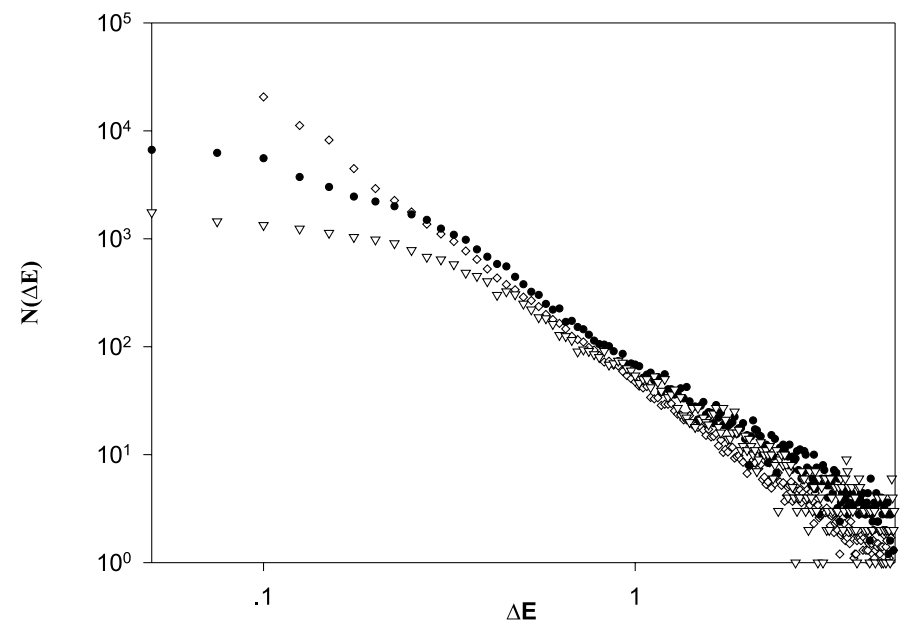

Figure 3

Statistics of energy release $N(\Delta E) . \diamond \mathrm{GMF}$ model, $N=10,000$ for 5000 samples, the slope is nearly -2.10 , - LMSC model, $N=40,000, \delta=5$, for 2000 samples, the slope is -2.34 approximately, $\nabla$ CMF model, $N=100,000$, for 3000 samples, the slope is about -2.59 .

transition in the model. The region surrounding the transition point may be considered the critical region.

From the viewpoint of rupture prediction, however, a power-law size-distribution might provide a negative conclusion (GELLER et al., 1997).

However, there is observational evidence, while indirect, of natural seismicity suggesting the existence of general features prior to the catastrophe transition or main rupture. To monitor these general features may provide clues for rupture prediction. There have been a number of reports of accelerating seismic moment release (AMR) (SAMmis et al., 1999; JAUMÉ et al., 1999) and a change in the rate of occurrence of moderate-sized earthquakes prior to large earthquakes in a variety of tectonic settings. Yin and co-workers (YIN et al., 1994) have reported numerous cases where a measure of the load-unload response ratio (LURR) has increased markedly prior to a number of moderate to large earthquakes. Recently, it was found that the optimal region size for AMR and for LURR is comparable, which may suggest that AMR and LURR have a common physical mechanism (YIN et al., 2002). These phenomena are considered to be evidence supporting Critical Point Hypothesis (CPH) (BowmAN et al., 1998; TIAMPO et al., 2000) of earthquakes, which predicts the existence of a critical region with the progressive formation of long-range stress field correlation, a condition required for large earthquakes. From our model, we also found general features related to catastrophe transition or main rupture. They are called critical sensitivity and trans-scale fluctuations, which will be discussed in section 4 and section 5 , separately. We will point out that there might be an essential relationship between $\mathrm{CPH}$ and catastrophe transition. In fact, the time 
series of energy release shown in Figure 1 displays an acceleration prior to catastrophe transition or main rupture. This might be similar to the AMR in earthquakes.

\section{Critical Sensitivity}

We report here a novel discovery of a general feature of systems in the vicinity of the catastrophe transition. This is called critical sensitivity, which means that the sensitivity of a system may strongly be enhanced in various aspects as the system approaches the catastrophe transition. In this section, we discuss two kinds of critical sensitivity, i.e., sensitivity to external loading and sensitivity to stochastic microdamage.

There is evidence to suggest that the earth's crust displays critical sensitivity prior to large earthquakes. Seismologists agree that foreshocks are a symptom of some preparatory process prior to the main rupture in some cases (WYSS et al., 1997; YIN et al., 1994). Yin et al. proposed a promising method for earthquake forecasting called the load-unload response ratio (LURR) (YIN et al., 1994, 1999; WANG et al., 1998). LURR is defined as the ratio of activity of small-to-intermediate earthquakes during a loading phase to the activity during an unloading phase, where the loading and unloading result from earth tides. It is found that in most cases (more than $80 \%$ ), LURR increases significantly before large earthquakes and fluctuates slightly about unity in stable regions.

The LURR method may be explained as follows. A large earthquake can be considered as a local catastrophic rupture in the earth's crust, and the increase in LURR prior to large earthquakes implies that a region of the crust has become significantly sensitive to external loading perturbation prior to catastrophe. Thus, we presume that the increase of LURR might be evidence for critical sensitivity prior to large earthquakes.

In order to explore the features of critical sensitivity, we performed simulations based on the coupled pattern mapping model.

The sensitivity of energy release to external loading can be measured by

$$
S=\frac{\Delta E^{\prime}}{\Delta \sigma_{0}^{\prime}} / \frac{\Delta E}{\Delta \sigma_{0}},
$$

where $\Delta E^{\prime}(\Delta E)$ is the energy release induced by increment $\Delta \sigma_{0}^{\prime}\left(\Delta \sigma_{0}\right)$, and

$$
\Delta \sigma_{0}^{\prime}=\Delta \sigma_{0}+\alpha \bar{\sigma}_{c}
$$

where $\bar{\sigma}_{c}$ is the average strength of units (the mean of the distribution function $h\left(\sigma_{c}\right)$ ) and $\alpha$ is a small parameter $\left(\alpha \sim 10^{-2} \rightarrow 10^{-3}\right)$. According to the definition of the sensitivity, $S>>1$ indicates high sensitivity, and $S \sim 1$ implies an insensitive state. 
The sensitivity of energy release to external loading is shown in Figure 4. Figure 4(a) shows the time series of $S$ for a sample. At the initial stage, $S$ maintains a low value (of order 1) but $S$ increases significantly near the catastrophe transition point $p / p_{c}=1$. Figures 4(b), (c) and (d) show the time series of $S$ for 200 samples identical macroscopically based on SRD models of GMF, LMSC and CMF, respectively. We can see that, although normalized variable $p / p_{c}$ is adopted, the time series of $S$ are different from sample to sample, which is evidence for sample-specificity. However, in all cases there is a common trend that $S$ increases significantly near the catastrophe transition. This is the hallmark of critical sensitivity.

Figure 5 shows ensemble statistics of maximum $S$ and the statistics of $p_{M} / p_{c}$, where $p_{M}$ is the damage fraction at which $S$ takes the maximum value $S_{\max }$. It is found that, for most samples, the sensitivity $S$ arrives at its maximum when $0.7 \leq p / p_{c} \leq 1$, and $S_{\max }$ is usually one order of magnitude higher than the initial value $S \approx 1$. These results imply that critical sensitivity is a significant precursor of the catastrophe transition.

Now we examine the sensitivity of energy release to stochastic microdamage. We consider a model where deterministic dynamics given by the coupled patten mapping and the stochastic microdamage coexist (XIA et al., 1996). The stochastic microdamage is modelled by the break of $\Delta n$ units chosen randomly at the beginning of a loading step. The sensitivity of energy release to the stochastic microdamage can be measured by the ratio of energy release with a stochastic microdamage to that without the stochastic damage:

$$
S^{*}=\frac{\Delta E^{*} / \Delta n}{\Delta E}
$$

where $\Delta E$ and $\Delta E^{*}$ are energy releases under the identical external loading condition but without and with stochastic microdamage of size $\Delta n$, respectively. Figure 6 shows $S^{*}$ for one sample and for 200 samples identical macroscopically. It is obvious that, like the behavior of $S, S^{*}$ also displays the sample-specificity and critical sensitivity.

It is interesting to note that the critical sensitivity of energy release to stochastic microdamage is a sensitivity linking different scales: from mesoscopic events to macroscopic behavior. Such a sensitivity implies that a minor change on the mesoscopic level can be strongly amplified during nonlinear evolution and leads to significant macroscopic effect as the system approaches the catastrophe transition. In section 3, we represent sample-specificity, i.e., samples which are identical macroscopically but different from sample to sample mesoscopically due to disordered meso-heterogeneity display diverse behavior of catastrophe transition. Samplespecificity is also a feature linking different scales: the macroscopic behavior is sensitively dependent on the details of mesoscopic structure. There is a common underlying physical mechanism behind sample-specificity and sensitivity of energy 


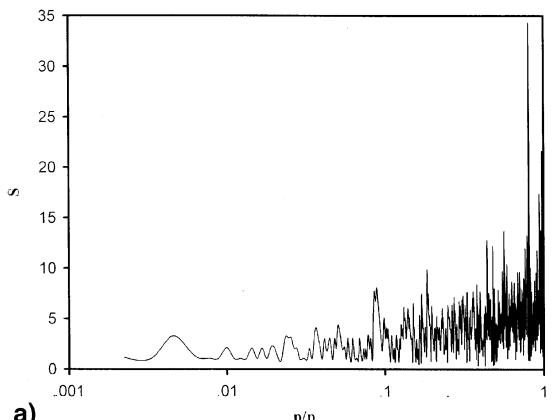

a)

$\mathrm{p} / \mathrm{p}_{\mathrm{c}}$

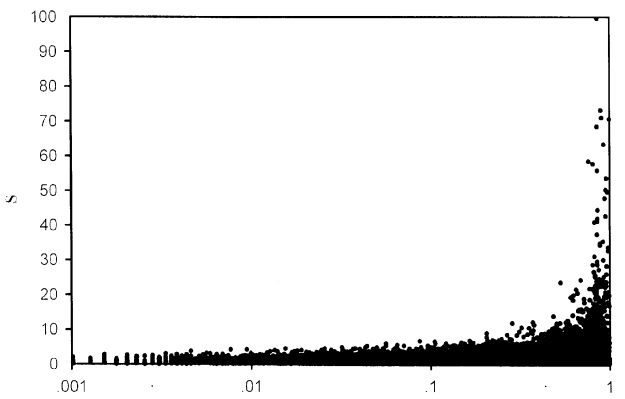

b)

$\mathbf{p} / \mathbf{p}$

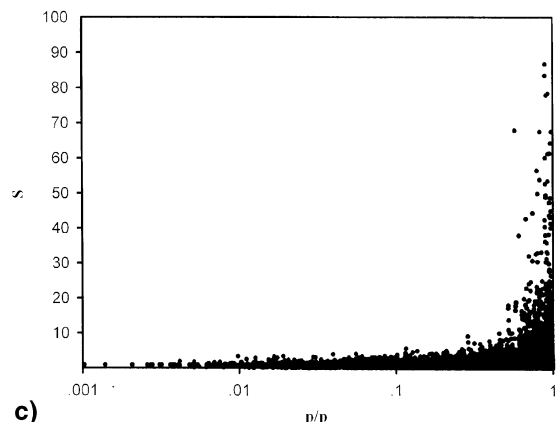

c)

$\mathrm{p} / \mathrm{p}$

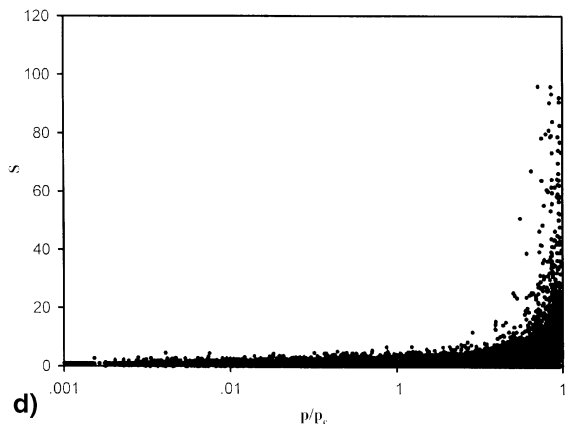

Figure 4

Time series of $S$, the critical sensitivity to external load pertubations $(N=10,000, \alpha=0.001)$. (a) for a single sample, CMF model, (b) for 200 samples, GMF model, (c) for 200 samples, LMSC model with $\delta=5$, (d) for 200 samples, CMF model. 

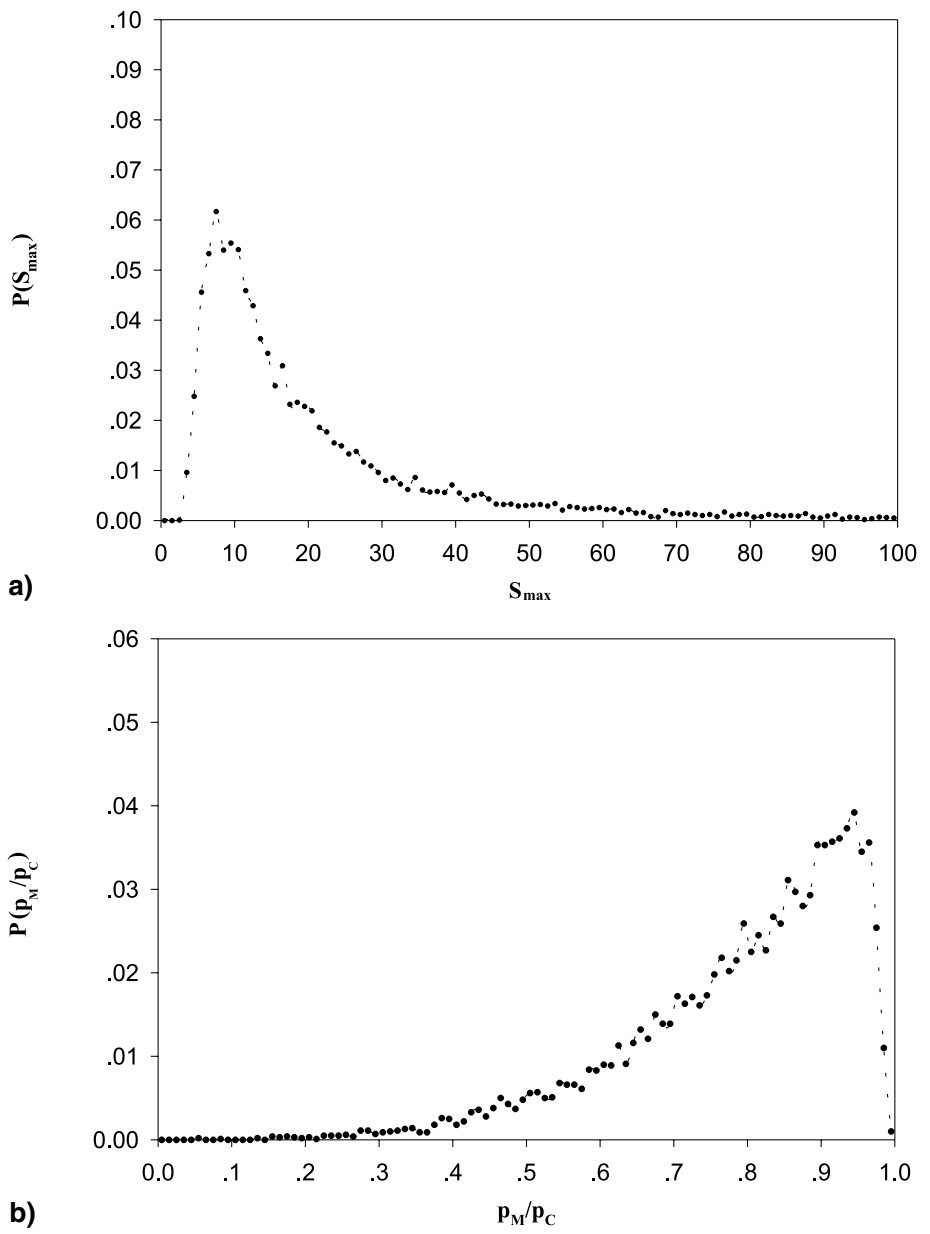

Figure 5

Ensemble statistics of (a) $S_{\max }$ and (b) $p_{M} / p_{c}$, for the CMF model with $N=4000$ and $\alpha=0.001$ (1000 samples).

release to stochastic microdamage. Such critical sensitivity is termed as trans-scale sensitivity.

The coupled pattern mapping model can be thought of driven nonlinear threshold systems, comprised of multitudes interacting, mesoscopic units subjected to a driving force. As the system approaches the catastrophe transition, increasingly more units are close to their threshold. It will be considerably easier to trigger larger cascade by perturbation at that time. This is the origin of critical sensitivity. Thus, critical sensitivity might be a general feature in widely used driven nonlinear threshold models. It is especially interesting to examine whether $\mathrm{CPH}$ also displays critical sensitivity. 

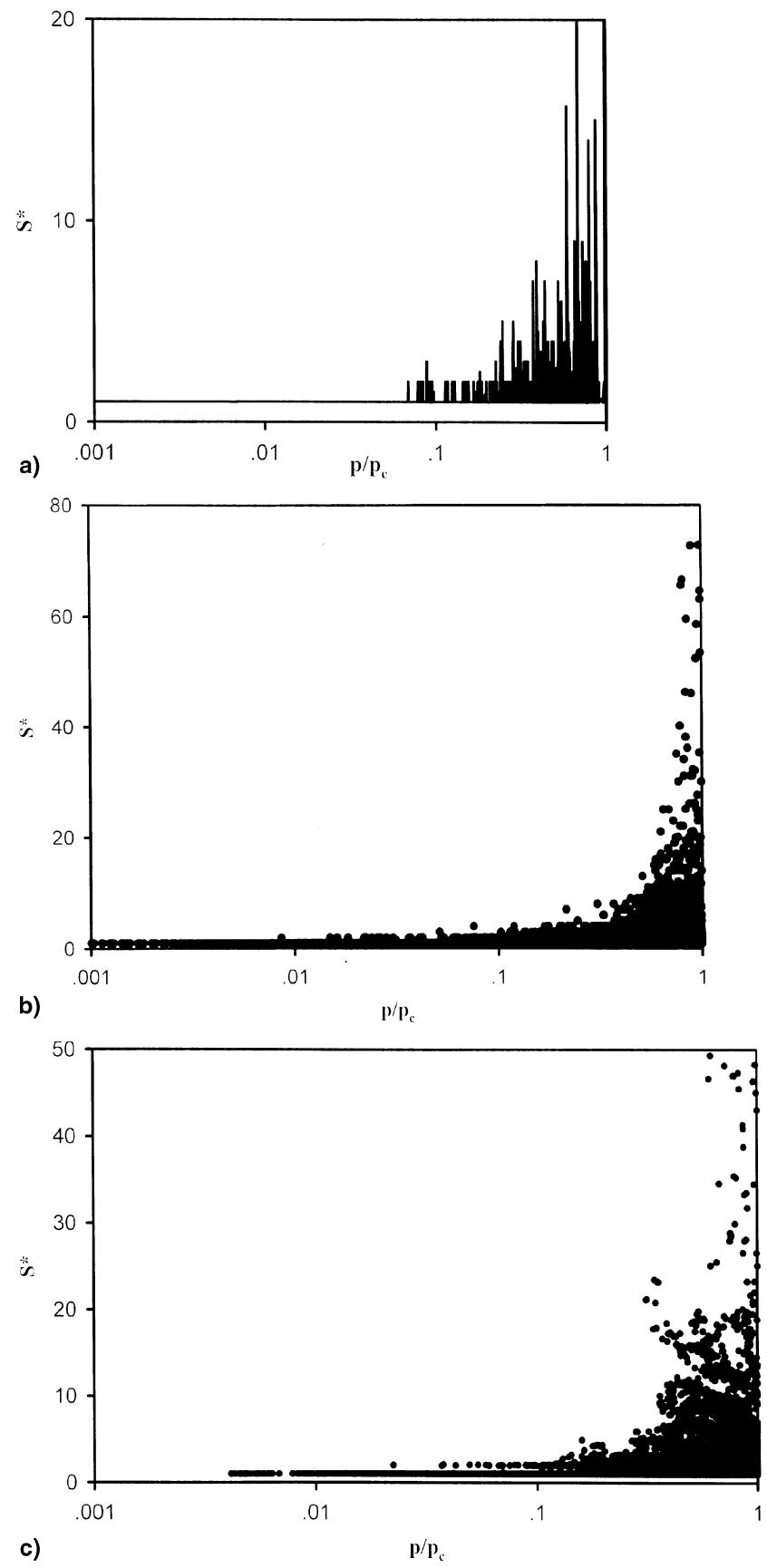

Figure 6

Time series of $S^{*}$, the critical sensitivity to stochastic microdamage $(N=10,000, \Delta n=1)$, (a) for a single sample, GMF model, (b) for 200 samples, GMF model, (c) for 200 samples, CMF model. 


\section{Trans-scale Fluctuations}

It is well known that fluctuations play a vital role in catastrophic rupture of heterogeneous brittle media. The rupture behavior of a system may not be completely representable by only its average macroscopic properties (XIA et al., 1997, 2000; IbNabdelualil et al., 1997; Wei et al., 2000; SAhimi et al., 1993). However, the general features of fluctuations related to catastrophe is still an open question. We report here a class of fluctuations related to catastrophe. At catastrophe threshold $\left(\sigma_{0}=\sigma_{0 f}\right)$ the stress fluctuations in system are enhanced significantly and the spatial scale of stress and damage fluctuations increases rapidly from the mesoscopic heterogeneity scale to the macroscopic scale; such fluctuations are called trans-scale fluctuations.

Stress fluctuations can be measured as the relative deviation of stress $\delta \sigma / \bar{\sigma}$, where

$$
\delta \sigma=\left[\frac{1}{N(1-p)} \sum_{i=0}^{N}\left(\sigma_{i}-\bar{\sigma}\right)^{2}\left(1-x_{i}\right)\right]^{1 / 2}
$$

is standard deviation of stress supported by intact units, and

$$
\bar{\sigma}=\frac{\sigma_{0}}{1-p}
$$

is mean stress on intact units. Figure 7 shows $\delta \sigma / \bar{\sigma}$ versus $p$, corresponding to the time-series of the relative deviation of stress fluctuations. It is also found that time series of $\delta \sigma / \bar{\sigma}$ shows sample-specificity, i.e., the time-series are different from sample to sample for samples which are identical macroscopically. However, the time-series display a general trend that $\delta \sigma / \bar{\sigma}$ increases rapidly beyond the transition point $p_{c}$ (keeping $\sigma_{0}=\sigma_{0 f}$ ) but prior to the main rupture. Ensemble statistics illustrate that, at the catastrophe threshold $\delta \sigma / \bar{\sigma}$ increases by about two orders of magnitude. This can be seen from the statistical distribution of maximum $\delta \sigma / \bar{\sigma}$ during GS regime and the catastrophe regime (shown in Fig. 8).

In order to reveal the characteristics of the stress pattern, we take a coarsegrained average of the stress pattern and examine its fluctuations. The approach is as follows: The system is divided into $m$ cells with size $C$, then $C=N / m$. Denote $\langle\sigma\rangle_{j}$ to be the average stress over intact units in the $j$-th cell, and $\langle\sigma\rangle_{j}=0$ if all units in the $j$-th cell are broken. Define the local damage fraction of the $j$-th cell as

$$
P_{j}=\frac{1}{C} \sum_{i \in j} x_{i}
$$

where the summation is over all units in the $j$-th cell. The relative deviation of coarsegrained average stress can be calculated using 

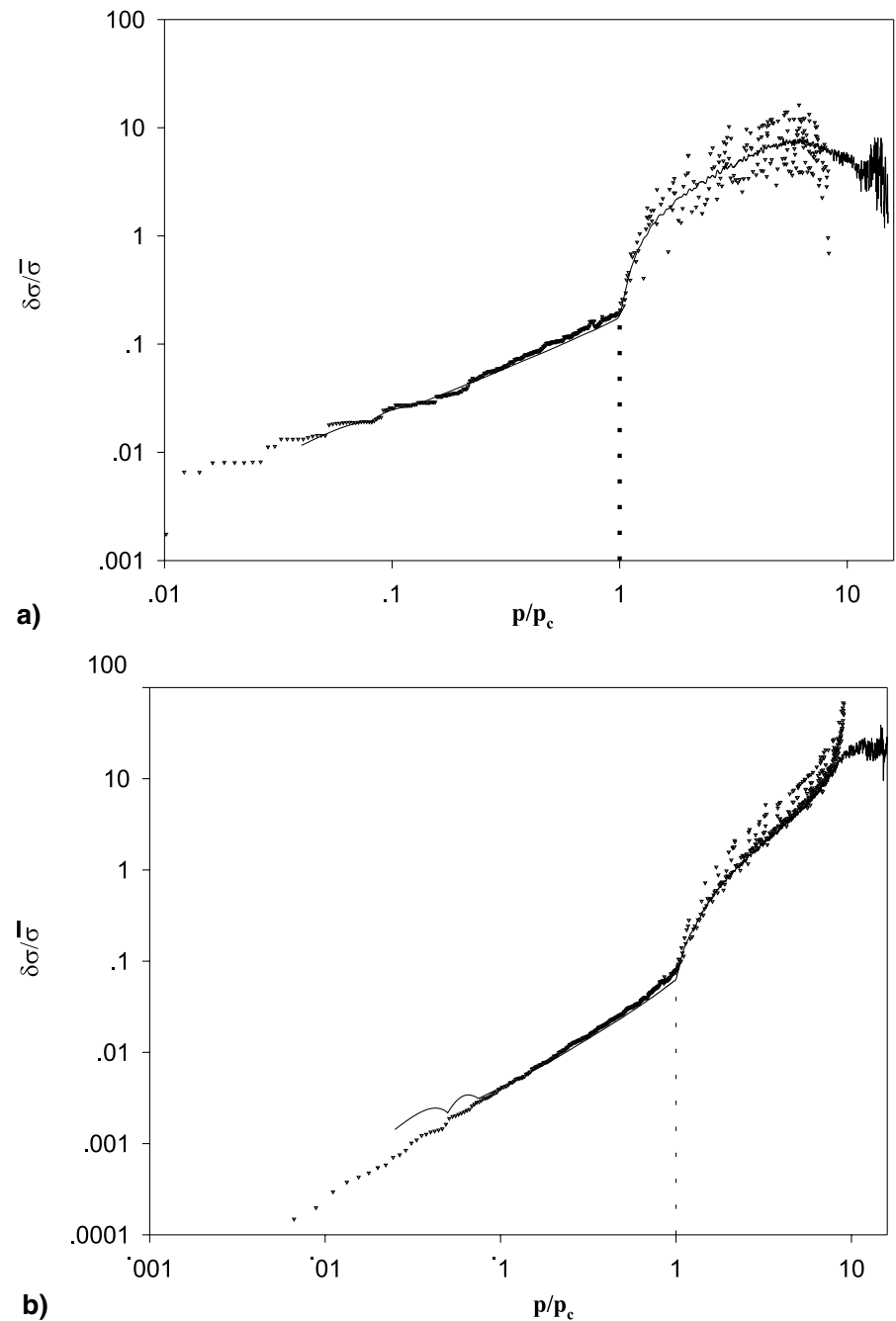

Figure 7

Relative deviation of stress fluctuations $\delta \sigma / \bar{\sigma}$ versus damage fraction $p(N=4096)$. The solid line is averaged over 200 samples while the triangle represents a single sample. (a) CMF model and (b) LMSC model with $\delta=5$.

$$
\frac{\delta\langle\sigma\rangle}{\bar{\sigma}}=\frac{1}{\bar{\sigma}}\left[\sum_{j} H\left(1-P_{j}\right)\left(\langle\sigma\rangle_{j}-\bar{\sigma}\right)^{2} / \sum_{j} H\left(1-P_{j}\right)\right]^{1 / 2}
$$

where $\bar{\sigma}$ is mean stress supported by intact units (given in Equation 5.2), and 


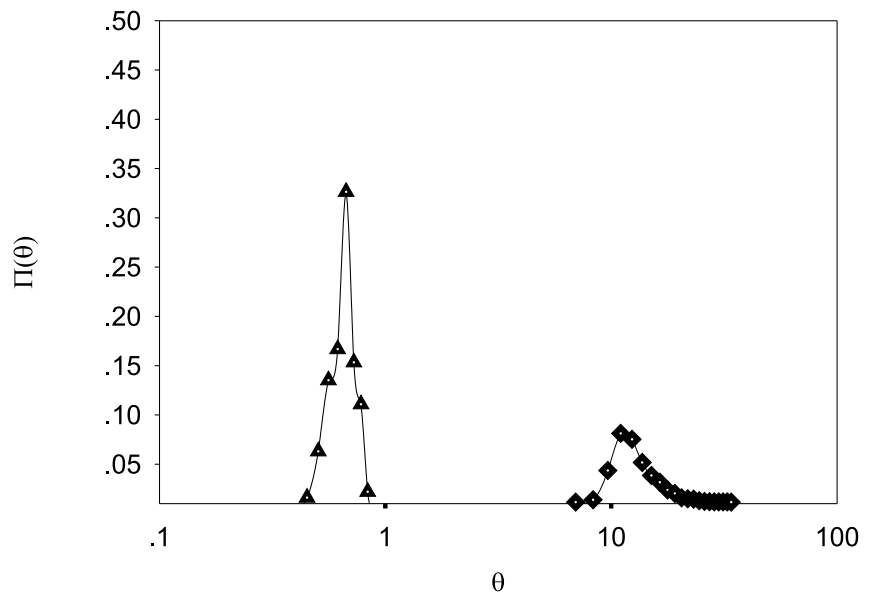

Figure 8

Ensemble distribution function (10,000 samples) of maximum values of $\delta \sigma / \bar{\sigma}$ for GS regime and catastrophic rupture regime $(N=20,000, \mathrm{CMF}$ model), The two distribution functions are well-separated, indicating that stress fluctuations increase significantly in the catastrophe regime.

$$
H(y)=\left\{\begin{array}{ll}
0, & \text { for } y=0 \\
1, & \text { for } y>0
\end{array} .\right.
$$

The relative deviation of fluctuations in the coarse-grained average stress is shown in Figure 9(a). A distinct feature is that, for the GS regime, $\delta\langle\sigma\rangle / \bar{\sigma}$ decreases significantly with increasing coarse-grained scale $C$ and $\delta\langle\sigma\rangle / \bar{\sigma} \approx 0$ for the macroscopic scale $C$. Qualitatively, stress pattern is macroscopically homogeneous in GS regime. At catastrophe threshold, macroscopic inhomogeneity of the stress pattern increases significantly.

The fluctuations of coarse-grained damage fraction $\left\{P_{j}, j=1,2, \ldots, m\right\}$ can be calculated by

$$
\delta P=\left[\frac{1}{m} \sum_{j=1}^{m}\left(P_{j}-p\right)^{2}\right]^{1 / 2},
$$

where $P_{j}$ and $p$ are defined in Equations (5.3) and (2.1), respectively. $\delta P$ is shown in Figure 9(b).

In reality, between the catastrophe transition and main rupture, there is a timeinterval, which may vary for different real systems. Trans-scale fluctuations occur during this interval and can be considered a significant indication of the catastrophe transition and an immediate precursor to main rupture. This may provide clues for prediction of the main rupture. The trans-scale fluctuations identified in the numerical simulations were compared to the statistics of damage events according to 

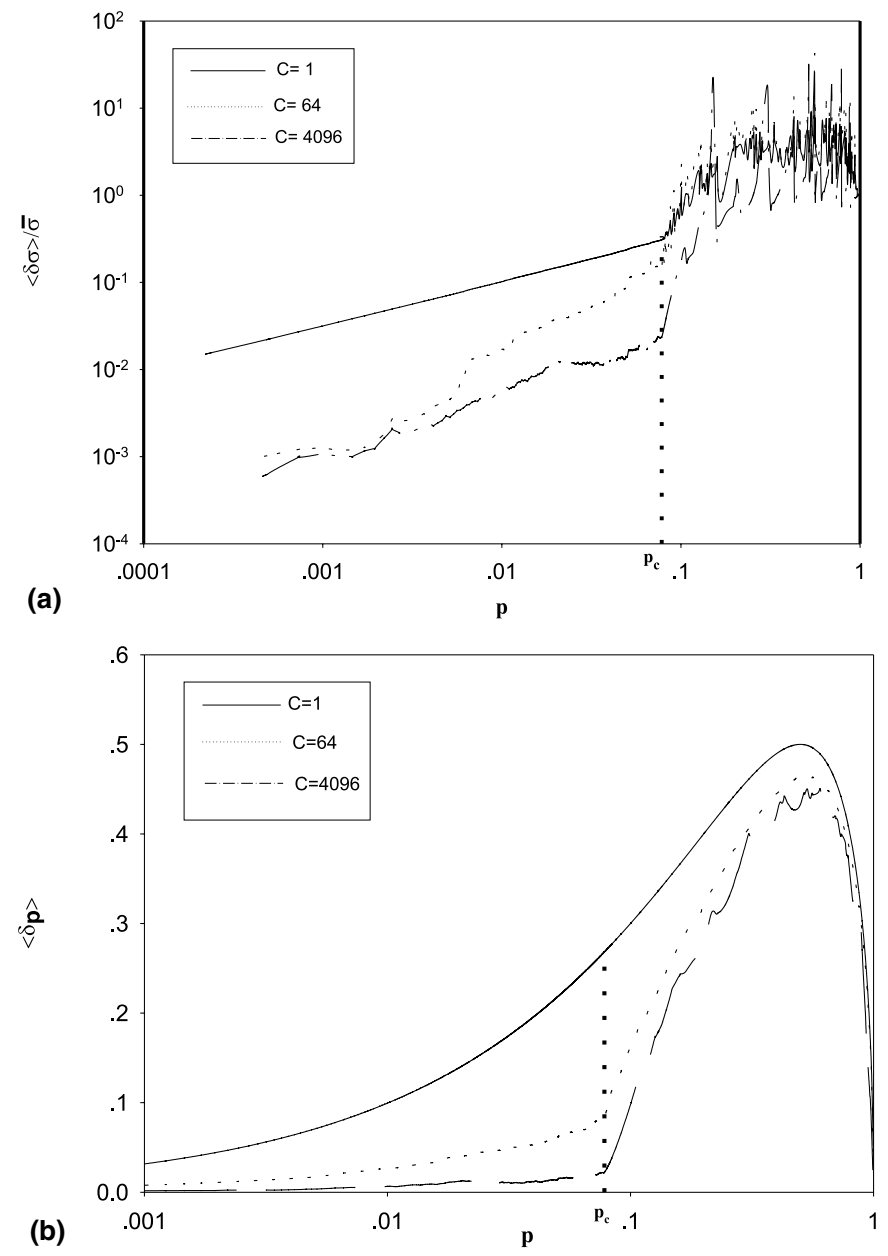

Figure 9

Trans-scale fluctuations: (a) the fluctuations of coarse-grained average stress $\langle\delta \sigma\rangle / \bar{\sigma}$ and (b) the fluctuations of coarse-grained average damage $\delta\langle p\rangle$ versus damage fraction $p$ (CMF models, $N=65,536$ ). The line type indicates the coarse-grained cell size $C$ : the solid line represnts $C=1$, the dotted line represents $C=64$, and the dashed line represents $C=4096$.

acoustic emission signals detected during the rupture process (LOCKNER et al., 1991, 1992; GARCIMARTIN et al., 1997).

Trans-scale fluctuations of stress imply that the correlation length of stress increases progressively from small scale to large scale at the catastrophe threshold. Long-range correlation of high stress is the condition required for main rupture to occur. This is very similar to the progressive formation of long-range stress field correlation predicted by Critical Point Hypothesis (CPH). 


\section{Summary and Discussions}

Rupture in heterogeneous brittle media was examined based on a model called a coupled pattern mapping model. The rupture presents dynamical complexity, especially the catastrophe transition and sample-specificity. This complexity is responsible for the difficulty of rupture prediction. However, we have found clues for rupture prediciton from general features of catastrophic rupture: critical sensitivity and trans-scale fluctuations. These may be viewed as precursors of catastrophe transition and main rupture.

The underlying mechanism of the catastrophe transition, sample-specificity, critical sensitivity and trans-scale fluctuations, should be attributed to the coupling between heterogeneity and dynamical nonlinearity, which leads to a cascade of damage. The cascade is determined by coupling, competition and balance between strength heterogeneity and stress redistribution (SRD). In the globally stable (GS) regime, a cascade induced by SRD is limited to finite size by surrounding stronger units. Beyond catastrophe transition however, the cascade can no longer be inhibited and will continue without limit, i.e., the system evolves to an entirely failed state. Critical sensitivity implies that, near the catastrophe transition, the size of the cascade can be enhanced significantly by minor external stress perturbations. This is because, near catastrophe, more and more units support stresses close to their strength, and thus, a small disturbance may induce significant extra failure of events. In the catastrophe regime, the cascade evolves from the mesoscopic scale to the macroscopic scale, resulting in trans-scale fluctuations.

The coupling between heterogeneity and dynamical nonlinearity leads to transscale sensitivity resulting in sample-specificity. This makes the problem far more complex. Consequently, to explore the general features of the catastrophe transition is a problem of great importance. Although critical sensitivity and trans-scale fluctuations are features of a simple conceptual model, we are quite sure that they present general features of a class of real systems, including perhaps the earth's crust.

Our work suggests that there is an essential relationship between the catastrophe transition and the $\mathrm{CPH}$. They display similar general features and might be attributed to a common underlying physical mechanism. A further work to compare the major features of the two theories would be very interesting.

\section{Acknowledgments}

This work is supported by the Special Funds for Major State Basic Research Project G2000077305 and National Natural Science Foundation of China (Grant No. 19732060, 19972004, 10047006). 


\section{REFERENCES}

Bak, P., TAng, C., and Wiesenfeld, K. (1987), Self-Organized Criticality: An Explanation of 1/f Noise, Phys. Rev. Lett. 59, 381-384.

BAK, P., TANG, C., and Wiesenfeld, K. (1988), Self-Organized Criticality, Phys. Rev. A 38, 364-374.

BAK, P. Self-Organized Criticality: A Holistic view of Nature. In Complexity: Metaphors, Models, and Reality (eds. Cowan, G., Pines, D. and Meltzer, D.) (SFI Studies in Sciences of Complexity, Proc. Vol. XIX, Addison-Wesley 1994) pp. 477-493.

BAI, Y. L., Lu, C.S., KE, F. J., XIA, M. F. (1994), Evolution Induced Catastrophe, Phys. Lett. A 185, 196199.

Ben-Zion, Y. and Sammis, C. G. (2001), Characterization of Fault Zones, Pure Appl. Geophys., (in press).

Bowman, D. D., Oulllon, G., Sammis, C. G., Sornette, A., and Sornette, D. (1998), An Observational Test of the Critical Earthquake Concept, J. Geophys. Res. 103, 359.

Curran, D. R., Seaman, L., and Shockey, D. A. (1997), Dynamic Failure of Solids, Phys. Rep. 147, 253388.

Curtin, W. A. (1997), Toughening in Disordered Brittle Materials, Phys. Rev. B 55, 11,270-11,276.

Coleman, B. D. (1958), On The Strength of Classical Fibers and Fiber Bundles, J. Mech. Phys. Solids 7, 6070.

Daniels, H. E. (1945), The Statistical Theory of The Strength of Bundles of Threads, Proc. Roy. Soc. London A 183, 405-435.

Diodati, P., Marchesoni, F., and Piazza, S. (1991), Acoustic Emission from Volcanic Rocks: An Example of Self-organize Criticality, Phys. Rev. Lett. 67, 2239-2242.

Garcimartin, A., Guarino, L., Bellon and Ciliberto, S. (1997), Statistical Properties of Fracture Precursors, Phys. Rev. Lett. 79, 3202-3205.

Gutenberg, B. and Richter, C. F. (1944), Frequency of Earthquake in California, Bull. Seismol. Soc. Am. $34,125-188$.

Geller, R. J., Jacksom, D. D., Kagan, Y. Y., and Mulargia, F. (1997), Earthquakes Cannot be Predicted, Science 275, 1616-1617.

Heimpel, M. (1997), Critical Behaviour and the Evolution of Fault Strength during Earthquake Cycles, Nature, 388, 865-868.

Ibnabdeljalil, M. and Curtin, W.A. (1997), Strength and Reliability of Fiber-reinforced Composites: Local Load Sharing and Associated Size Effects, Int. J. Solids and Structures 34, 2649-2668.

Jaumé, S. C. and Sykes L. R. (1999), Evolving Towards a Critical Point: A Review of Accelerating Seismic Moment/Energy Release Prior to Large and Great Earthquakes, Pure Appl. Geophys. 155, $279-305$.

Knopoff, L. (2000), The Magnitude Distribution of Declustered Earthquakes in Southern California, Proc. Natl. Acad. Sci. USA 97, 11,880-11,884.

Lockner, D. A., Byerlee, J. D., Ponomarev, A., and Sidorin, A. (1991), Quasi-Static Fault Growth and Shear Fracture Energy in Granite, Nature 350, 439-443.

Lockner, D. A. and Byerlee, J. D., Precursory AE Patterns Leading to Roch Fracture. In Proc. 5th Conf. on Acoustic Emmisions/Microseismic Active in Geologic Structure and Materials, (ed. Hardy, H.R.) (Trans. Publ. 1992) pp. 1-4.

Lu, C. S., Takayasu, H., Tretyakov, A. Y., Takayasu, M., and Syumoyo, S. (1998), Lattice Model of the Brittle Crust, Self-Organized Criticality in a Block, Phys. Lett. A 242, 349-354.

Langer, J. S., Carlson, J. M., Myers, C. R., and Shaw, B. E. (1996), Slip Complexity in Dynamic Models of Earthquake Faults, Proc. Natl. Acad. Sci. USA 93, 3825-3829.

Meakin, P. (1991), Models for Material Failure and Deformation, Science 252, 226-234.

Sahimi, M. and Arbabi, S. (1993), Mechanics of Disordered Solid. III. Fracture Properties, Phys. Rev. B 47, $713-722$.

Sammis, C. G. and Smith S. W. (1999), Seismic Cycles and the Evolution of Stress Correlation in Cellular Automaton Models of Finite Fault Networks, Pure Appl. Geophys. 155, 307-334.

Stein, R. S. (1999), The Role of Stress Transfer in Earthquake Occurrence, Nature 402, 605-609.

Swinbanks, D. (1997), Quake Panel Admits Prediction is 'Difficult', Nature 388, 4. 
Tiampo, K. F., Rundle, J. B., Gross, S. J., and Klein, K. (2000), Karhunen-Loeve Expansion Analysis of Seismicity on the Southern California Fault System, EOS Trans. AGU, 81(48), Fall Meet. Suppl., Abstract NG61A-12, 2000.

Turcotte, D. L. (1999), The Physics of Earthquakes: Is It a Statistical Problem?, In Proc. 1-st ACES Workshop Proceedings. (ed. Mora, P.), 95-98 (The APEC Cooperation for Earthquake Simulation, Brisbane).

Wang, C. Y. and CAI, Y. E. (1997), Sensitivity of Earthquake Cycles on the San Andreas Fault to Small Changes in Regional Compression, Nature 388, 158-161.

WAnG, Y. C., YIN, X. C., and WANG, H. T. (1998), The Experimental Simulation of Rocks on Load/Unload Response Ratio for Earthquake Prediction, Earthquake Research in China (English Version) 12, 367-372.

WeI, Y. J., XIA, M. F., KE, F. J., YIN, X. C., and BAI, Y. L. (2000), Evolution-Induced Catastrophe and Its Predictability, Pure Appl. Geophys. 157, 1945-1957.

Wyss, M., Aceves, R. L., Park, S. K., Geller, R. J., Jackson, D.D., Kagan, Y.Y., and Mulargia F. (1997), Cannot Earthquakes Be Predicted?, Science 275, 487-490.

XIA, M. F., BAI, Y. L., and Ke, F. J. (1996), A Stochastic Jump and Deterministic Dynamics Model of Impact Failure Evolution with Rate Effect, Theor. Appl. Frac. Mech. 24, 189-196.

XIA, M. F., KE, F. J., BAI, J., and BAI, Y. L. (1997), Threshold Diversity and Trans-Scales Sensitivity in a Nonlinear Evolution Model, Phys. Lett. A 236, 60-64.

XIA, M. F., Ke, F. J., WeI, Y. J., BAI, J., and BAI, Y. L. (2000), Evolution Induced Catastrophe in a Nonlinear Dynamical Model of Materials Failures, Nonlinear Dynamics 22, 205-224.

Yin, X. C., Chen, X. Z., Song, Z. P., and Yin, C. (1994), A New Approach to Earthquake Prediction: Load/Unload Response Ratio (LURR) Theory, Pure Appl. Geophys. 145, 701-705.

Yin, X. C., Chen, X. Z., Wang, Y. C., Wang, H. T., Peng, K. Y., Zhang, Y. X., and Zhuang, J. C. (1999), Development of a New Approach for Earthquake Prediction - The Load/Unload Response Ratio. In Proc. 1-st ACES Workshop Proceedings. (ed. Mora, P.), pp. 325-330 (The APEC Cooperation for Earthquake Simulation, Brisbane).

Yin, X. C., Mora, P., Peng, K. Y., Wang, Y. C., and Weatherly, D. (2002), Load-unload Response Ratio and Accelerating Moment/Energy Release, Critical Region Scaling and Earthquake Prediction, Pure appl. geophys. 159, 2511-2523.

(Received February 20, 2001, revised June 11, 2001, accepted June 25, 2001)

To access this journal online:
(20) http://www.birkhauser.ch

